\title{
On Capturing Spatio-temporal Factors in Cognitive Network Channel Selection
}

\author{
Joshal Daftari, Bheemarjuna Reddy Tamma, B. S. Manoj, and Ramesh Rao \\ California Institute for Telecommunications and Information Technology - UC San Diego, USA \\ E-mail: \{jdaftari, btamma, bsmanoj, rrao $\} @$ ucsd.edu
}

\begin{abstract}
In this paper, we present an application of probabilistic graphical models such as Bayesian Networks (BNs) for capturing the spatio-temporal factors in cognitive networks. We propose to use a BN that makes use of historical network information to learn the network behavior across spatio-temporalspectral dimensions and predicts best configuration for each Access Point (AP) in a Wireless LAN (WLAN) system. We further present the application of BNs for traffic prediction as well as channel selection in a cognitive WLAN scenario. Our results prove that the space and time are critical factors that can impact the performance of the network configuration. We noticed improvement in traffic prediction accuracy and channel selection accuracy, respectively, of $35 \%$ and $40 \%$, when using space and time information.
\end{abstract}

\section{INTRODUCTION}

In a Wireless LAN (WLAN), Access points (APs) are specially configured wireless devices that are connected to a wired network and act as central transmitters and receivers of radio signals. Due to the dynamic and shared nature of wireless medium, parameters controlling access to the wireless medium on each AP must be monitored frequently and modified in a coordinated fashion which is a laborious task if done manually. To maximize WLAN performance, autonomic network control which involves automation of the network reconfiguration process is highly essential. The concept of cognitive network was described in [1] as a network that can observe the current network conditions and plan, decide, and act for future network conditions while maintaining the endto-end objectives. The key contribution of this paper is the use of a probabilistic graphical model, Bayesian Network (BN), to learn network behavior across spatio-temporal-spectral dimensions and predict best configuration for each AP in a WLAN system.

\section{RELATED WORK}

Cognitive networking, a relatively new paradigm, follows the evolution of the cognitive radio systems. Examples of endto-end and system-wide cognitive networking approaches can be found in [1]. To the best of our knowledge, there exist only limited works that exploit graphical models in the context of networking systems [2], [3]. In [2], a BN is used to diagnose TCP connection failures in Overlay networks. In [3], the authors looked at the problem of finding most suitable radio access technology for network reconfiguration in infrastructure based heterogeneous wireless networks at a very abstract level by considering only a few parameters.
Compared to the existing work, we focus on designing cognitive controller system for WLANs which can achieve an optimal network configuration based on the probabilistic dependency relationship derived using a variety of network and environmental parameters such as traffic parameters, channel errors, space, time, and spectrum. To accomplish our objective, we take the help of BN models which is briefly reviewed next.

\section{A. Overview of Bayesian Networks}

A BN, widely used for statistical inference and machine learning [4], is a representation of statistical relationships between random variables through a Directed Acyclic Graph (DAG). A node of this graph represents a random variable and the presence of an edge between two nodes represents a direct probabilistic relation between them. The BN lends itself with several advantages like encoding dependencies among network parameters, learning causal relations among parameters and hence serves as an ideal representation for combining prior knowledge with historical dataset representing the network behavior. The main stages in developing and using a BN model are as follows.

1) Structure Learning: The goal of structure learning is to learn a DAG that best explains a given dataset using heuristic approaches like constraint-based and search-and-score techniques. Constraint-based algorithms (e.g., PC algorithm) usually start with a fully connected graph, and progressively remove edges connecting the variables if certain conditional independencies are measured in the historical data. In the more commonly employed search-and-score approaches (e.g., greedy Hill Climbing (HC) [5] and K2 algorithm [6]), the main task is a search, through the space of possible DAGs, which is intended to return one or a set of possible sample BN structures. A structure with maximum score is selected using popular structure scoring metrics like Bayesian Information Criterion (BIC) which incorporate a penalty for complexity to guard against over-fitting of data.

2) Parameter Learning: This involves learning parameters of conditional probability distributions once the structure is known. It is carried out using existing algorithms such as maximum likelihood estimation (MLE) or the principle of maximum entropy.

3) Inference: Since BNs represent joint probability distributions of the parameters of interest, we could use them to compute other related probabilities through inference methods which mainly include belief updating and belief revision (or 


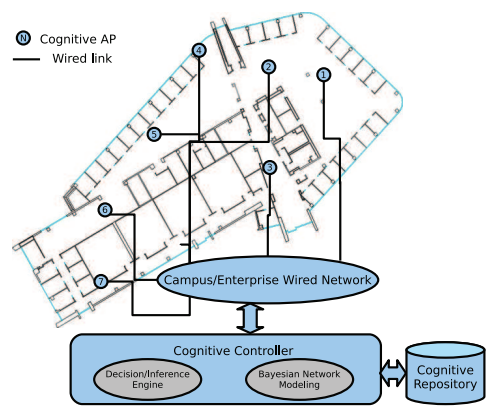

Fig. 1. Cognitive Network Architecture.

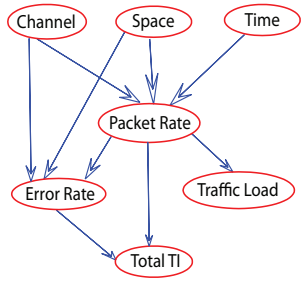

(a)

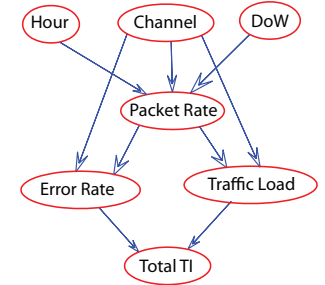

(b)

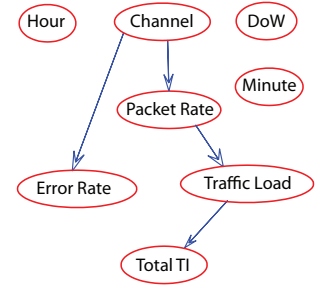

(c)
Fig. 2. BN model created by taking (a) 28 weeks of real network data from several CogNet APs (b) 2 weeks of historical data from an autonomous CogNet AP (c) 6 hours of data from a field-deployed CogNet AP during the Operation Golden Eagle emergency response drill. abductive inference). In belief updating, some of the nodes in the graph are clamped to evidence values, and we wish to compute posterior distributions of one or more subsets of other nodes. Abductive inference has two variants: Most Probable Explanation (MPE) which finds the most probable configuration of all nodes given evidence nodes and Maximum A posteriori (MAP) which finds the most probable configuration of a subset of nodes (typically the controllable network parameters) given evidence nodes.

\section{OUR WORK}

\section{A. Cognitive Network Architecture}

The cognitive network architecture for enterprise/campus WLAN system is shown in Figure 1. A collection of Cognitive APs (CogNet APs) forms the basis of our system. To gather the traffic information from multiple channels, an additional network interface dedicated for traffic sensing is used. Each CogNet AP is connected to the central cognitive controller through a wired link and remits all the traffic related measurement samples to the central cognitive database repository. Through this historical information, the central controller realizes cognitive functionality by constructing Bayesian structure that represents probabilistic relationships among network parameters and infers optimal values for controllable parameters such as operating channel based on current network conditions with the help of Decision/Inference Engine Module. It is worth to highlight that central cognitive controller can offload the decision making tasks to individual CogNet APs for achieving autonomous functionality at APs.

\section{B. Bayesian Modeling of WLAN System}

An example BN structure that we derived from real network parameters collected over a period of 28 weeks in our academic campus using several CogNet APs is shown in Figure 2(a). In this example, we have considered seven parameters: network parameters like Channel $(\mathrm{CH})$, Packet arrival Rate (PR), Error Rate (ER, number of CRC/PHY errors reported by the wireless card), Traffic Load (TL, in bps) and Total Traffic Intensity (TTI, takes into account the fraction of time in which the channel is occupied due to wireless transmissions as well as channel bandwidth consumed by $\mathrm{CRC} / \mathrm{PHY}$ error packets) and environmental parameters such as Space (S, location of AP) and Time (T, measurement time).
The edge from $\mathrm{CH}$ to PR indicates that the operating Channel influences the observed Packet Rate. This is true because in ISM band the orthogonal channels $(1,6$, and 11 for IEEE $802.11 \mathrm{~b} / \mathrm{g}$ WLANs) face more traffic than other channels. Similarly, the edge from $S$ to $P R$ is justified as traffic pattern is quite different at different $\operatorname{CogNet}$ AP locations.

As mentioned in Section III-A, the CogNet APs can function autonomously with the centralized cognitive controller running within the device. To demonstrate this, we consider the BN structures shown in Figures 2(b) and 2(c) which are learned by an autonomous CogNet AP in different environments. The former is derived using network data collected over a period of 2 weeks at our office building whereas later is obtained from the Operation Golden Eagle drill conducted in San Marcos city during May 2010. Time parameters such as Minute (MT, taken only for the short duration event in Figure 2(c)), Hour (HR) and binary DayOfWeek (DoW, takes value 1 for weekend and 2 for weekday) are considered along with network parameters already defined above. Out of the network parameters, Channel is the only directly controllable parameter. To simplify the structure learning process, we applied range discretization technique and mapped each continuous variable PR, ER, TL and TTI in the scale from 1 to $10 . \mathrm{CH}, \mathrm{MT}, \mathrm{HR}$ and DoW take a value in the range: 1 to 11,1 to 60,1 to 24 , and 1 to 2 , respectively. Using the Bayesian Net Toolbox (BNT) for Matlab [7], we generated Bayesian graphs for most of the popular structural learning algorithms. The structures used for this study are obtained using $\mathrm{HC}$ search-and-score technique which is initialized with a DAG given by $\mathrm{K} 2$ algorithm. The scoring method used is BIC. For parameter learning, we applied the Maximum Likelihood Estimation (MLE) method.

Several interesting conditional dependencies are revealed by observing Figures 2(b) and 2(c). For instance, from the former we find that given PR, the variables $\mathrm{HR}$ and TL are conditionally independent whereas in the later we observe that the network structure is independent of time. This difference is because the scenario in Figure 2(c) represents a short duration event. These results show that our model is quite accurate in capturing the dependencies between network and spatiotemporal parameters. Using such relations, we develop our framework for predicting the traffic and selecting the optimal channel. 


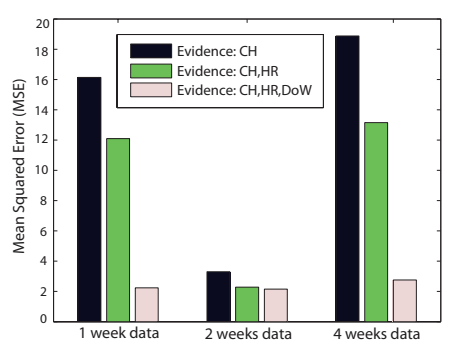

(a)

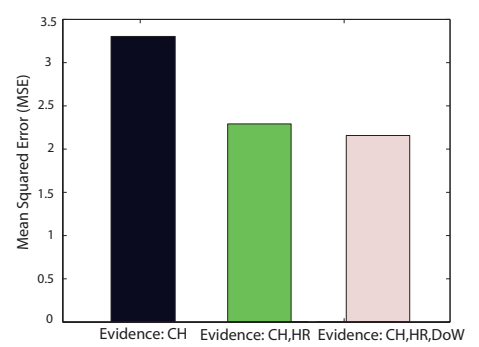

(b)

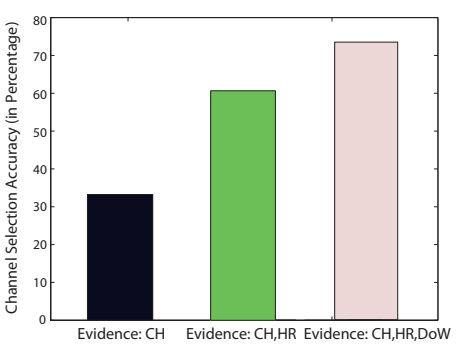

(c)

Fig. 3. For an autonomous Cognet AP: (a) Traffic prediction accuracy for different sizes of learning datasets (b) Traffic prediction accuracy for different evidence sets (c) Channel selection accuracy for different evidence sets.

\section{Performance Results}

We now illustrate the real benefits of Bayesian modeling by employing the $\mathrm{BN}$ model learned from our office environment for the following applications.

\section{A. Traffic Prediction in WLANs}

Here we show how the time for learning the structure as well as evidence of different network parameters affect accuracy of traffic prediction. We took TTI as our channel resource consumption metric because it captures the traffic load due to successful and errored packets. We studied its prediction accuracy using MAP which can be formulated as [8]:

$$
T_{\text {pred }}(\mathbf{M}, \mathbf{e}) \stackrel{\text { def }}{=} \underset{\mathbf{m}}{\arg \max } \operatorname{Pr}(\mathbf{m}, \mathbf{e})
$$

where $\mathbf{m}$ is an instantiation of the subset of parameters $\mathbf{M}$ (for our case $\mathbf{m} \in\{\mathrm{TTI}\}$ ), $\mathbf{e}$ is the instantiation of an evidence set (here $\mathbf{e} \in\{\mathrm{S}, \mathrm{C}, \mathrm{HR}, \mathrm{DoW}\}$, where $\mathrm{S}$ is constant for a given location), $\operatorname{Pr}(\mathbf{m}, \mathbf{e})$ is the joint probability of $\mathbf{m}$ and $\mathbf{e}$ and $T_{\text {pred }}$ is the predicted TTI value. Figure 3(a) shows mean squared error (MSE) between predicted and real (target) traffic values of structures learned for the period of 1,2 , and 4 weeks. From this figure it is observed that the MSE is minimal when we use historical data for 2 weeks. This makes our strategy very useful in residential and enterprise environments where the CogNet APs can quickly reach optimal configuration. Therefore, we have chosen Figure 2(b) as our ideal structure for this CogNet AP. Further, for the rest of the results, we use a history of 2 weeks data.

From Figure 3(b), it can be observed that additional evidence (in this case, Hour and DayOfWeek) to the initial evidence (Channel) helps in reducing the MSE by 35\% for the parameter under study.

\section{B. Channel Selection Accuracy}

For channel selection, if we assume that the optimal channel is the one that has minimum TTI, we can use the predicted TTI values obtained for each Channel using Equation 1 to find the estimated best channel which can be given by $C_{\text {est }}(\mathbf{h})=\arg \min T_{\text {pred }}(\mathbf{m}, \mathbf{h})$ where $C_{\text {est }}$ is the estimated best channel and $\mathbf{h} \in\{\mathrm{S}, \mathrm{HR}, \mathrm{DoW}\}$. Given $C_{\text {est }}$, we can compute accuracy of channel selection using a metric called Channel Selection Accuracy (CSA) which is the number of times (expressed as percentage) $C_{\text {est }}$ equals the actual channel with minimum TTI, $C_{a c t}$, for different evidence sets in the test dataset. CSA can be expressed as :

$\mathrm{CSA}=\frac{\sum_{\mathbf{i} \in \mathbf{B}}\{A(\mathbf{i})\}}{|\mathbf{B}|} \times 100 ; A(\mathbf{i})=\left\{\begin{array}{l}1: \text { if } C_{\text {est }}(\mathbf{i})=C_{\text {act }}(\mathbf{i}) \\ 0: \text { otherwise }\end{array}\right.$

where $\mathbf{B}$ is the set of evidence sets in the test dataset and $|\mathbf{B}|$ is the number of elements in B. Figure 3(c) shows that additional evidence increases the CSA by $40 \%$ and helps in better channel selection.

\section{SUMMARY}

In this work, we presented a Bayesian Network (BN) based strategy for capturing spatio-temporal factors in a cognitive wireless network. Further, we extend our BN-based model for accurate channel selection as a function of space and time. The use of additional evidences, DayofWeek and Hour, improved the traffic prediction and channel selection accuracies by approximately $35 \%$ and $40 \%$, respectively.

\section{ACKNOWLEDGEMENT}

This work is supported in part by the U. S. Army Research Office under grant number: W911NF-09-1-0456, the Center for Wireless Communications (CWC), and the WIISARDSAGE Project.

\section{REFERENCES}

[1] R. W. Thomas, D. H. Friend, L. A. DaSilva, and A. B. MacKenzie, "Cognitive networks: Adaptation and learning to achieve end-to-end performance objectives," IEEE Communications Magazine, vol. 44, no. 12, pp. 51-57, December 2006.

[2] G. J. Lee and L. Poole, "Diagnosis of tcp overlay connection failures using bayesian networks," pp. 305-310, 2006.

[3] G. Dimitrakopoulos, K. Tsagkaris, K. Demestichas, E. Adamopoulou, and P. Demestichas, "A management scheme for distributed cross-layer reconfigurations in the context of cognitive b3g infrastructures," Computer Communications, vol. 20, no. 18, pp. 3807-3822, December 2007.

[4] D. Heckerman, "A tutorial on learning with bayesian networks," Microsoft Research, Technical Report MSR-TR-95-06, March 1995.

[5] D. Chickering, "Optimal structure identification with greedy search," Machine Learning Research, vol. 3, pp. 507-554, 2002.

[6] G. F. Cooper and E. Herskovits, "A bayesian method for the induction of probabilistic networks from data," Machine Learning, vol. 9, no. 4, pp. 309-347, October 1992.

[7] K. Murphy, "Bayes Net toolbox for Matlab," Available: code.google.com/ $\mathrm{p} / \mathrm{bnt} /$.

[8] A. Darwiche, Modeling and Reasoning with Bayesian Networks. Cambridge University Press, 2009. 